\title{
Diagnostic d'un syndrome de Cowden à partir de manifestations cutanéo-muqueuses
}

\section{Diagnosis of a Cowden's syndrome with cutaneo-mucosis manifestations}

\author{
CÉDRIC HUARD, LOÏC ANTOINE, LAURENT DEVOIZE, CHRISTOPHE DESCHAUMES,
} THIERRY ORLIAGUET, MARTINE BAUDET-POMMEL

\section{RÉSUMÉ}

Une femme de 34 ans a consulté pour des délabrements et des mobilités dentaires. L'examen clinique a mis en évidence la présence de nombreuses lésions de la muqueuse linguale et d'une dizaine de lésions cutanées. Cette patiente avait été suivie pendant de nombreuses années pour des angiomes, des polypes gastro-intestinaux, des tumeurs ovariennes et thyrö̈diennes, et des kystes multiples sans qu'un diagnostic n'ait été réellement posé, malgré des manifestations caractéristiques d'un syndrome de Cowden. Med Buccale Chir Buccale 2007; 13: 87-91.

mots clés: Cowden, hamartomes multiples, cancers, langue scrotale

\section{SUMMARY}

A 34 year old woman consulted for oral problems. The clinical examination highlighted the presence of many lesions of the lingual mucous membrane and ten cutaneous lesions. This patient was followed during many years for gastro-intestinal angiomas, polyps, ovarian and thyroid tumours, and multiple cysts without a diagnosis being really posed, in spite of demonstrations characteristic of a syndrome of Cowden. Med Buccale Chir Buccale 2007; 13: 87-91.

key words: Cowden, multiple hamartomas, oncology, stone tongue

* Inserm E216, Université Clermont I, 11 boulevard Charles de Gaulle, 63000 Clermont-Ferrand

** Faculté de Chirurgie dentaire, Sous-section Médecine buccale - Chirurgie buccale, Université Clermont I,

11 Boulevard Charles de Gaulle, 63000 Clermont-Ferrand

*** Service d'Odontologie, Sous-section Médecine buccale - Chirurgie buccale, CHU, 63000 Clermont-Ferrand

Demande de tirés à part:

Martine Baudet-Pommel Faculté de Chirurgie dentaire Sous-section Médecine buccale - Chirurgie buccale Université Clermont I

11 boulevard Charles de Gaulle 63000 Clermont-Ferrand martine.baudet@u-clermont1.fr 
médecine buccale chirurgie buccale

VOL. $13, \mathrm{~N}^{\circ} 2$ 2007

page 88
Le syndrome de Cowden est une génodermatose rare, à transmission autosomique dominante, associée à diverses lésions tumorales, bénignes ou malignes. II est aussi appelés syndrome des "hamartomes multiples "; ils sont d'origine ectodermique, endodermique ou mésodermique, et affectent différents tissus ou systèmes. II comporte également des angiomes muqueux et cutanés, des lésions micro-kystiques, des cancers du poumon, des goitres thyroïdiens, des cancers de la thyroïde, des polypes angiomateux du tractus gastro-intestinal.

Le dépistage précoce d'un syndrome de Cowden est important pour anticiper le développement éventuel d'une tumeur maligne. Le cas présenté a été diagnostiqué tardivement ; chaque manifestation clinique ayant été considérée de façon indépendante malgré la présence des éléments caractéristiques classiques de ce syndrome.

\section{OBSERVATION}

En 1997, une femme de 34 ans consulte pour des délabrements et des mobilités dentaires nécessitant plusieurs avulsions. A l'interrogatoire, elle signale qu'elle a été opérée en 1962 à l'âge de quelques mois pour une verrue au genou droit. En 1974, elle a été hospitalisée pour un angiome à la jambe gauche qui a été traité par sclérose. En raison de l'extension de la lésion, une nouvelle intervention a été nécessaire. Au cours des trois années suivantes, la patiente s'est plainte de douleurs permanentes dans la jambe opérée. En 1978, la patiente consulte son médecin généraliste pour des douleurs abdomino-pelviennes. II évoque le diagnostic de coliques hépatiques ou néphrétiques. En 1981, son dentiste traitant pour une hypertrophie gingivale, couleur lie de vin, une affection parodontale et un bruxisme. Des prélèvements buccaux ont été réalisés mais il n'a pas été possible de retrouver les résultats. En 1987, en dermatologie, pour des lésions cutanées pour lesquelles aucun renseignement n'a pu être obtenu. En 1991, devant la persistance des douleurs pelviennes, un examen gynécologique a été réalisé : il met en évidence des lésions kystiques dans l'ovaire gauche et l'examen anatomo-pathologique révèle un cancer de l'ovaire. Une ovario- hystérectomie est pratiquée, suivie d'une chimiothérapie qui a due être interrompue à la troisième séance en raison d'une réaction d'intolérance. Quatre mois plus tard on décèle la présence de polypes dans l'estomac ; deux séances de laser ont été réalisées au centre anti-cancéreux. En 1992, un kyste se développe sur le pied droit. En 1993, la patiente consulte pour une " glossite » qui a été traitée par laser. En 1996, de nouveaux kystes sont apparus sur le pouce, le pied et l'avant-bras. Trois nodules thyroïdiens sont découverts ; une thyroïdectomie est réalisée. Au même moment, une importante perte de calcium et des crises tétaniques sont survenues.

En 1997, lors de l'examen de la patiente, on observe l'existence d'une dizaine de papules cutanées fermes, sessiles, rosées dans les régions périorbitaires, périorale et naso-géniennes (Fig. 1) pour lesquelles un traitement à base d'isotrétinoïne a été prescrit. La patiente présente également une glossite avec langue saburrale, des lésions en forme de pavés (type " cobblestones ") (Fig. 2 et 3), et des mobilités dentaires nécessitant l'avulsion de quelques dents (15, 17, 18, 28 et 36). Après plusieurs années d'évolution de la maladie, le diagnostic de syndrome de Cowden est évoqué et confirmé par le service de médecine interne. En 2004, cette patiente a été revue dans le service de chirurgie vasculaire après l'apparition de nouveaux angiomes.

\section{COMMENTAIRES}

Le syndrome de Cowden est une affection souvent mal connue bien qu'ayant fait l'objet de plusieurs publications. Dans $95 \%$ des cas, il intéresse la population caucasienne et affecte plus volontiers les sujets de sexe féminin (60\%) ${ }^{[1]}$. Les symptômes apparaissent généralement entre la trentième et la quarantième années ${ }^{[1,2,3]}$. La majorité des nouveaux cas diagnostiqués surviennent isolément, sans aucuns antécédents familiaux ${ }^{[4]}$. Toutefois Carlson ${ }^{[5]}$ signale que dans 10 à $20 \%$ des cas, un autre membre de la famille présente cette affection. Environ $80 \%$ des patients ayant un syndrome de Cowden ont une manifestation dermatologique. Les lésions cutanées se présentent sous forme de papules papil- 


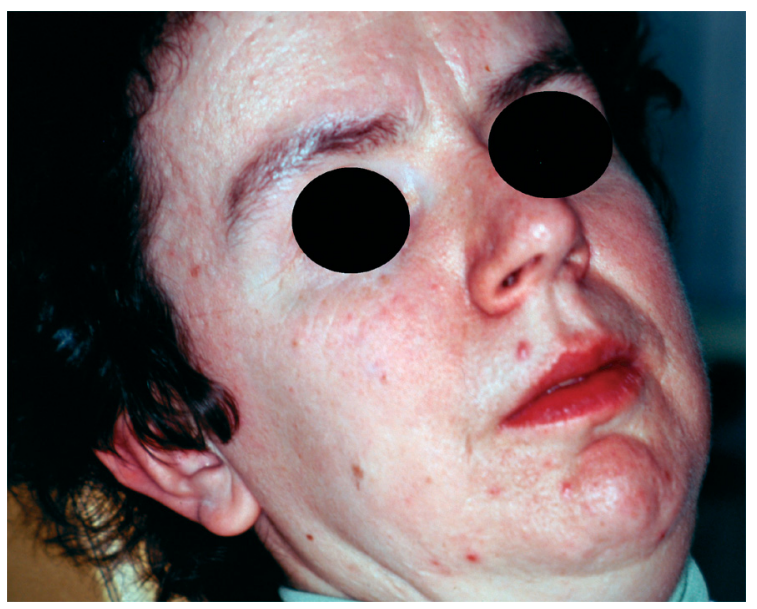

Figure 1 : Papules cutanées fermes, sessiles, rosées dans les régions péri-orbitaire, péri-buccale et nasogénienne

Cutaneous papulous strong, sessile, dew of the periorbital, peri-oral area and jugal

lomateuses ou lichénoïdes réparties sur la tête, le cou, les bras et les avant-bras. Dans la région maxillofaciale, c'est essentiellement autour de la bouche, du nez et dans la région périorbitaire que les papules sont retrouvées [3,4]. Histopathologiquement, ces lésions sont identifiées comme des tricholemmomes ou hamartomes dérivés des follicules pileux [6,7]. Ils constituent la manifestation la plus fréquente. Les plages kératosiques, formant des papules lisses ou rugueuses, siégeant sur le dos des mains et des pieds, viennent en second rang. D'autres lésions telles qu'un vitiligo, des taches café au lait et des mélanomes ont été décrites [7].

L'atteinte des muqueuses gingivales et buccales, avec présence de lésions papuleuses fibromateuses, est pathognomonique. Bien que les localisations électives soient les lèvres, la langue, les gencives, le rebord alvéolaire et la muqueuse jugale, d'autres zones comme le palais, la luette, l'oro-pharynx, le larynx et la muqueuse nasale peuvent être touchées. Les lésions buccales, en forme de pavés ou " cobblestones », sont rencontrées chez $40 \%$ des patients ${ }^{[3]}$.

Parmi les manifestations extracutanées, présentes chez 2/3 des patients, on retrouve une atteinte thyroïdienne qui se manifeste par un goitre, un dysfonctionnement thyroïdien, une thyroïdite ou un cancer de la thyroïde dans $7 \%$ des cas. La

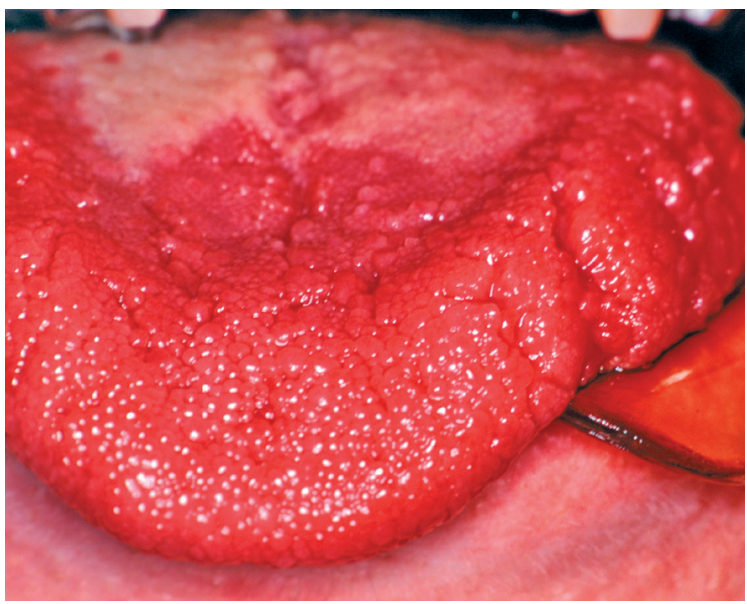

Figure 2 : Glossite avec langue saburrale scrotale et des lésions de type « cobblestones»

Glossite with sabbural scrotal tongue and cobblestones lesion

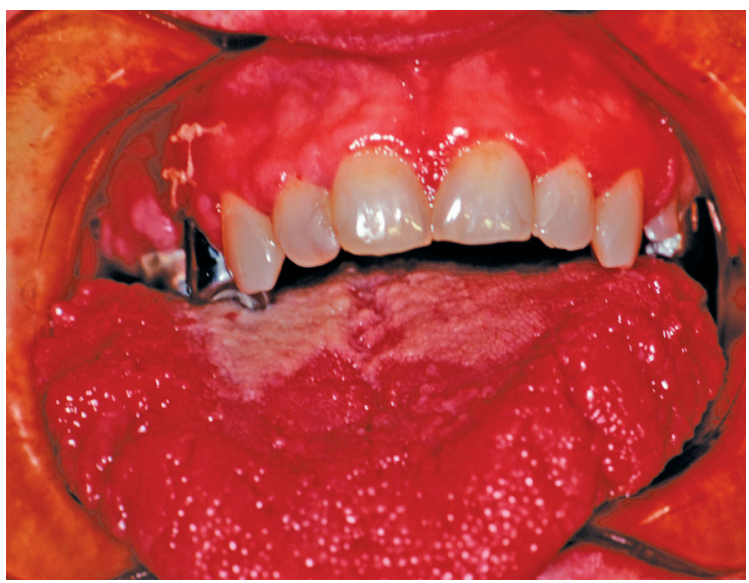

Figure 3 : Langue saburrale scrotale avec des lésions type cobblestones, associée à une hypertrophie gingivale

Saburral scrotal tongue with cobblestones lesions and hypertrophic gingivitis

thyroïde représente l'organe le plus touché après la peau.

Des fibrokystes et des fibroadénomes mammaires apparaissent chez $75 \%$ des sujets de sexe féminin ; quant au cancer du sein, il s'observe chez $22 \%$ des patientes à un âge comparable à celui de la population générale ${ }^{[4,8]}$.

Les polypes hamartomateux du tractus gastrointestinal, retrouvés chez $40 \%$ des patients, peuvent survenir en l'absence de toutes lésions cutanéo-muqueuses ${ }^{[8]}$.

Des mutations génétiques de la protéine PTEN (phosphatase et homologue de la tensine), gène médecine buccale chirurgie buccale VOL. $13, \mathrm{~N}^{\circ} 2$ 2007

page 89 
médecine buccale chirurgie buccale

VOL. $13, \mathrm{~N}^{\circ} 2$ 2007 page 90

Tableau 1 : Critères opérationnels internationaux pour le diagnostic du syndrome de Cowden (version 2000) ; selon Pilarski et Eng [12] International Cowden consortium operational criteria for the diagnostis of Cowden syndrome (version 2000); in Pilarski and Eng [12]

\begin{tabular}{lll} 
Critères pathognomoniques & Critères majeurs & Critères mineurs \\
\hline Lésions cutanéo-muqueuses & Cancer du sein & Goitre thyroïdien \\
Trichilemmomes faciaux & Cancer de la thyrö̈de & Retard mental \\
Kératoses & Macrocéphalie & Polypes intestinaux hamartomateux \\
Lésions papillomateuses & Maladie de Lhermitte-Duclos & Lipomes \\
Lésions muqueuses & Carcinome endométrial & Fibromes \\
& & Tumeurs génito-urinaires
\end{tabular}

Le diagnostic de certitude d'un syndrome de Cowden est fait lorsqu'au moins un de ces critères est retrouvé : 1. Lésions cutanéo-muqueuses pathognomoniques si : présence de six papules faciales ou plus, dont au moins trois doivent être un tricholemmome, ou des papules faciales cutanées et une papillomatose orale floride, ou une papillomatose buccale muqueuse et une kératose acrale, ou au moins six kératoses palmo-plantaires.

2. Deux critères majeurs dont un doit être la macrocéphalie ou la maladie de Lhermitte-Duclos.

3. Un critère majeur et trois critères mineurs.

4. Quatre critères mineurs.

Dans une famille où une personne est déjà atteinte d'un syndrome de Cowden, les critères diagnostiques sont modifiés et se présentent ainsi :

1. Lésions cutanéo-muqueuses pathognomoniques

2. Au moins un critère majeur avec ou sans critères mineurs

3. Deux critères mineurs

suppresseur de tumeur localisé en 10q23.3, ont été retrouvées dans $80 \%$ des cas; plus de 80 mutations différentes ont été identifiées. [9,10,11]. Les critères pathognomoniques de la maladie ont fait l'objet d'un consensus (Tab. 1), selon Pilarski et Eng [12]. Ils confirment bien que dans $90 \%$ des cas on retrouve des lésions cutanéo-muqueuses avec présence de tricholemmomes faciaux, de kératoses papillomateuses et de lésions muqueuses ${ }^{[2,3]}$.

\section{CONCLUSION}

Le syndrome de Cowden est une cause rare d'affections cutanées et des lésions fibro-papillomateuses de la cavité buccale. Le développement des lésions cutanéo-muqueuses précède souvent l'apparition des affections tumorales qui intéressent principalement le sein et la thyroïde.

La prise en charge de ce syndrome nécessite un diagnostic précoce et un suivi régulier des patients afin de dépister l'apparition de lésions malignes. Ce dépistage doit être étendu systématiquement à l'ensemble de la famille. Des mammographies périodiques sont recommandées ainsi que des bilans thyroïdiens (tomodensitométrie, examens de laboratoire...) et des contrôles gynécologiques répétés.

Le traitement des papules faciales fait appel à l'isotrétinoïne, au 5-fluoro-uracyl, ou au curetage chirurgical. Le traitement au laser ou l'excision chirurgicale peuvent aussi être indiquées. 


\section{RÉFÉRENCES}

1 - Capitan Canadas LM, Salinas Sanchez JL, Martinez Castillo SL, Labrot Moléon N, Duran Moreno D, Sanchez Lopez D, Valencia Laseca E. Multiple oral fibropapillomatosis as an initial manifestation of Cowden syndrom. Case Report. Med Oral Patol Oral Cir Bucal 2006 ; 11 : E319-24.

2 - Waite KA, Eng C. Protean PTEN : form and function. Am J Human Genet $2002 ; 70$ : 829.

3 - Olympia Kovich MD, Cohen D. Cowden's syndrome: Dermatology Online Journal $2004 ; 10: 3$.

4 - Hildenbrand C, Burgdorf WH, Lautenschlager S. Cowden syndrome : diagnostic skin signs. Dermatology $2001 ; 2002: 362$

5 - Carlson GJ, Nivatvongs S, Snover DC. Colorectal polyps in Cowden's disease (multiple hamartoma syndrom). Am J Surg Pathol $1984 ; 8$ : 763-70.

6 - Schreibman IR, Baker M, Amos C, Mc Garrity TJ. The hamartomatous polyposis syndroms: a clinical and molecular review. Am J Gastroenterol 2005 ; 100 : 47690.

7 - Thyresson HN, Doyle JA. : Cowden's disease (multiple hamartoma syndrom). Mayo Clin Proc 1981 ; 56 : 17984.

8 - McGarrity TJ, Wagner Baker MJ, Ruggiero FM, Thiboutot DM, Hampel H, Zhou XP, Heng C. Gl polyposis and glycogenic acanthosis of the esophagus associated with PTEN mutation positive Cowden syndrome in the absence of cutaneous manifestations. Am J Gastroenterol 2003 ; 98 : 1429.
9 - Bussaglia E, Pujol RM, Gil MJ, Marti RM, Tuneu A, Febrer Ml, Garcia-Patos V, Ruiz EM, Barnadas M, Alegre M, Serrano S, Matias-Guiu X. PTEN mutations in eight Spanish families and one Brazilian family with Cowden syndrome. J Invest Dermatol $2002 ; 118: 6$.

10 - Zhou XP, Waite KA, Pilarski R, Hampel $H$, Fernandez MJ, Bos C, Dasouki M, Fedman GL, Greenberg LA, Ivanovich J, Matloff E, Patterson A, Pierpont ME, Russo D, Nassif NT, Eng C. Germline PTEN promoter mutations and deletions in Cowden / Bannayan-Riley-Ruvalcaba syndrome result in aberrant PTEN protein and dysregulation of the phosphoinositol-3-kinase: Akt pathway. Am J Hum Genet 2003 ; 73 : 404

11 - Reifenberg J, Rauch L, Beckmann MW, Megahed N, Ruzicka T, Reifenberg G. Cowden's disease: clinical an molecular genetic finding in a patient with a novel PTEN germline mutation. Br J Dermatol 2003 ; 148 : 1040.

12 - Pilarski R, Eng C. Will the real Cowden syndrome please stand up (again)? Expanding mutational and clinical spectra of the PTEN hamartoma tumour syndrome. J Med Genet 2004 ; 41 : 323-6.

\section{médecine buccale chirurgie buccale}

VOL. $13, \mathrm{~N}^{\circ} 2$ 2007

page 91 